\title{
Exclusión Educativa de Personas con Discapacidad: Un Problema Pedagógico
}

\author{
Educational Exclusion of People with Disability: A Pedagogical \\ Problem
}

Pilar Cobeñas *

Universidad Nacional de La Plata, Argentina

\begin{abstract}
La educación inclusiva ha irrumpido en la agenda educativa global hace por lo menos tres décadas, produciendo que los sistemas educativos mundiales estén siendo revisados en torno a sus principios pedagógicos. Esta forma educativa surgió debido al reconocimiento en muchos países, incluida Argentina, de que los sistemas educativos estaban dejando a muchos niños y niñas fuera de la escuela o recibiendo una educación más pobre que el resto (Miles y Ainscow, 2008). El grupo de personas con discapacidad es uno de los grupos más perjudicados, por lo que, si bien la educación inclusiva hace referencia a todos los grupos marginados de los sistemas educativos, en este artículo nos ocuparemos especialmente de dicho colectivo. Se ha utilizado un método cualitativo con enfoque etnográfico que pone en el centro las narrativas de jóvenes con discapacidad en contexto escolar. El estudio se ha desarrollado en cuatro escuelas comunes y cuatro especiales de la región bonaerense de Argentina. En nuestro estudio pudimos identificar al menos tres grados de incumplimiento del derecho a la educación inclusiva en la escolarización de las personas con discapacidad: exclusión escolar, segregación e integración o exclusión en la inclusión. Asimismo, intentamos analizar los supuestos pedagógicos que atraviesan dichas formas educativas, tensionándolas con la perspectiva de educación inclusiva, comprendida tanto como una concepción pedagógica, así como un derecho.
\end{abstract}

Descriptores: Integración escolar; Discapacidad; Exclusión social; Segregación escolar.

Inclusive education burst onto the global educational agenda at least three decades ago, making the world's education systems to revise its pedagogical principles. This educational form arose due to the recognition in many countries, including Argentina, that the educational systems were leaving many children out of school or giving a poorer education than the rest (Miles and Ainscow, 2008). The group of people with disabilities is one of the most disadvantaged groups, so, although inclusive education refers to all marginalized groups of education systems, in this article we will take special care of this group. A qualitative method with an ethnographic approach has been used to put the narratives of young people with disability in the school context. The study has been developed in four regular schools and four special schools in the Buenos Aires region of Argentina. In our study, we were able to identify at least three degrees of non-compliance with the right to inclusive education in the schooling of people with disability: exclusion, segregation and integration or exclusion in inclusion. Likewise, we try to analyze the pedagogical assumptions that these educational forms go through, viewing them through the perspective of inclusive education, understood as much as a pedagogical conception, as well as a right.

Keywords: School integration; Disability; Social exclusion; School segregation.

*Contacto: pilarcobenas@gmail.com

ISSN: 1696-4713

www.rinace.net/reice/

revistas.uam.es/reice
Recibido: $\quad 1$ de abril 2019

$1^{\text {a }}$ Evaluación: 18 de mayo 2019

$2^{\text {a }}$ Evaluación: 7 de junio 2019

Aceptado: $\quad 12$ de junio 2019 


\section{Revisión de la literatura}

La educación inclusiva ha irrumpido en la agenda educativa global hace por lo menos tres décadas, produciendo que los sistemas educativos mundiales estén siendo revisados en torno a sus principios pedagógicos. Esta forma educativa surgió debido al reconocimiento en muchos países, incluida Argentina, de que los sistemas educativos estaban dejando a muchos niños y niñas fuera de la escuela o recibiendo una educación más pobre que el resto (Miles y Ainscow, 2008). El grupo de personas con discapacidad es uno de los grupos más perjudicados, por lo que, si bien la educación inclusiva hace referencia a todos los grupos marginados de los sistemas educativos, en este artículo nos ocuparemos especialmente de dicho colectivo.

Según las Naciones Unidas (ONU, 2013), de cuatro formas de escolarización que existen actualmente para las personas con discapacidad, tres resultan discriminatorias: la exclusión, la segregación y la integración. La educación inclusiva constituye la única forma educativa que efectiviza el derecho a la educación de las personas con discapacidad. En consecuencia, la educación inclusiva no solamente significa una perspectiva pedagógica, sino que también constituye un derecho. Así lo explica un documento de Naciones Unidas sobre el derecho a la educación inclusiva en las personas con discapacidad denominado "Estudio temático sobre el derecho de las personas con discapacidad a la educación. Informe de la Oficina del Alto Comisionado de las Naciones Unidas para los Derechos Humanos" (en adelante, “Estudio Temático"):

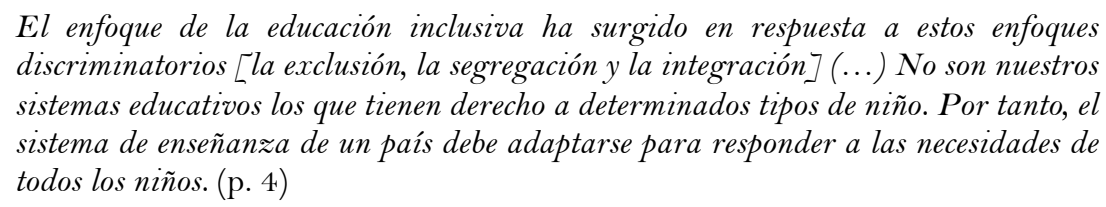
discriminatorios [la exclusión, la segregación y la integración] (...) No son nuestros sistemas educativos los que tienen derecho a determinados tipos de niño. Por tanto, el sistema de enseñanza de un país debe adaptarse para responder a las necesidades de todos los niños. (p. 4)

Tal como destaca el Estudio Temático, las personas con discapacidad son quienes tienen derecho a que las escuelas se transformen en pos de una educación inclusiva, y no las escuelas las que pueden decidir a qué alumnos educar.

Siguiendo las demandas del movimiento de personas con discapacidad, así como los aportes de los estudios sociales de la discapacidad, se parte de la convicción de que el pleno ejercicio del derecho a la educación de los grupos excluidos del sistema escolar, o segregados en instituciones "especiales", sólo puede darse por medio de una educación inclusiva que problematice esta categorización y tenga como objetivo una educación para todos y todas juntos/as (Cobeñas, 2016; Echeita y Ainscow, 2011). Así, se considera a la educación inclusiva no como un fin en sí misma,

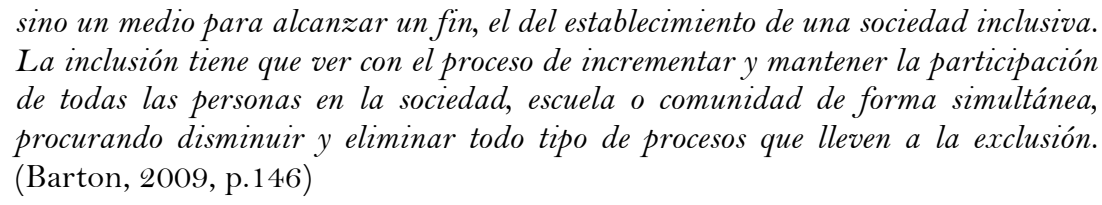

Estas demandas del colectivo de personas con discapacidad han conseguido, entre otros resultados, la firma de la Convención sobre los Derechos de las Personas con Discapacidad (ONU, 2006, de reciente jerarquía constitucional; en adelante "la Convención"), ratificada por la Argentina, y con actual jerarquía constitucional, que en su artículo 24 obliga al Estado Nacional a asegurar el derecho a la educación inclusiva de las personas con discapacidad. Pero esto requiere identificar barreras y construir condiciones institucionales y conocimiento didáctico que contribuya a efectivizar dicho derecho. 
Los debates sobre la educación de las personas con discapacidad se enmarcan en dos grandes paradigmas: el modelo médico y el modelo social. El paradigma del modelo médico produce una concepción de las personas con discapacidad como falladas o inferiores biológicamente. Dichas miradas implican que las diferencias en ciertos cuerpos y ciertas capacidades son comprendidas como disvalores. De modo que "ser discapacitado supone experimentar la discriminación, la vulnerabilidad y los asaltos abusivos a la propia identidad y estima” (Barton, 1998, p. 24). Esta perspectiva subyace a las múltiples prácticas de exclusión que van desde algunas formas de discriminación hasta el exterminio de este grupo social.

Mike Oliver, un reconocido teórico de la discapacidad recientemente fallecido, define a la teoría de la tragedia personal o "modelo individual" (2008, p. 20) como el principal obstáculo por el cual no se ha tenido en cuenta a la discapacidad en las políticas públicas y no se ha avanzado en términos académicos en un sentido sociológico y social. Desde su perspectiva, esta mirada tendría como consecuencia la creencia de que las personas con discapacidad no pueden participar plenamente de la sociedad debido a sus propias limitaciones físicas o psicológicas, y que éstas son generadas por la condición de persona con discapacidad vista como una situación vivida por la persona y su entorno como traumática.

Sin embargo, cada vez más personas con discapacidad señalan que no son las limitaciones personales las que evitan su plena participación, sino las restricciones sociales que la misma sociedad les impone lo que les lleva a adoptar la opinión de que "la discapacidad es, por lo tanto, una forma particular de opresión social" (Oliver, 2008, p. 20).

En esta línea, el movimiento de personas con discapacidad ha luchado desde sus inicios para revertir los sentidos negativos asociados a la discapacidad y construir una identidad positiva, al entender que "la discapacidad es una categoría social y política en cuanto implica prácticas de las regulaciones y las luchas por la posibilidad de elección, la potenciación y sus derechos" (Barnes, 1998, p. 24). Así, en articulación con los estudios sociales de la discapacidad se construyó el paradigma del modelo social, que comprende a la discapacidad como un efecto de las barreras que la sociedad impone sobre algunas personas con ciertas características (Barton, 2009b).

El modelo social ha tenido, asimismo, un gran valor en las vidas de las personas con discapacidad. En palabras de Liz Crow, una mujer con discapacidad, citada por Jenny Morris (1998):

\begin{abstract}
Desde hace varios años ya que este modelo social de la discapacidad me ha permitido confrontar, sobrevivir e incluso superar innumerables situaciones de exclusión y discriminación. Ha sido mi pilar, como ha sido para el movimiento de discapacidad más ampliamente. (...) Ha jugado un rol central en la promoción del auto valor de las personas con discapacidad, identidad colectiva y organización politica. No creo que sea una exageración decir que el modelo social ha salvado vidas. (p. 3, traducción propia)
\end{abstract}

De modo que podemos afirmar que modelo social de la discapacidad tiene un gran impacto ya que ha posibilitado a las personas con discapacidad salir del modelo de la tragedia personal para auto valorarse y además les ha servido para identificarse y agruparse, entre otros beneficios. Asimismo, ha tenido un gran impacto en cómo pueden y deben ser pensados los sistemas educativos y las formas de enseñanza. Así surge la educación inclusiva, desde una mirada pedagógica desde el modelo social (Barton, 2009a). 
Desde esta perspectiva es que, según los estudios académicos sobre educación inclusiva (por ejemplo, Ainscow, 1998, 2002; Barton, 1998; Echeita, 2008; Parrilla, 2002; Porter, 2008; Rappoport et al., 2019), documentos como el Index para la Inclusión (Booth et al., 2015) o el Estudio temático, se entiende explícitamente por educación inclusiva la incorporación de todo el alumnado, incluidas las personas con discapacidad, a la escuela común. Sin embargo, esta incorporación, para ser comprendida como inclusión debe ser comprendida de una forma particular: deben asegurarse una correcta planificación y disposición de recursos en pos de la construcción de espacios y comunidades educativas que aseguren la valoración y participación plena de todos sus miembros y de hacer efectivo el derecho a la educación en una lucha constante contra la exclusión.

En el marco del presente artículo nos proponemos comunicar algunos resultados de un estudio que tuvo como objetivo indagar acerca de los procesos de exclusión educativa que afectan a las personas con discapacidad en Argentina. En este sentido, se espera contribuir desde una perspectiva pedagógica a la identificación de barreras para la transformación del sistema educativo en inclusivo y visibilizar las formas específicas de exclusión que afectan a las personas con discapacidad, vulnerando su derecho a la educación.

\section{Método}

El siguiente artículo presenta algunos resultados obtenidos a partir de una investigación que consistió en problematizar los procesos de inclusión y exclusión escolar de jóvenes mujeres con discapacidad y personas con discapacidad en general en Argentina tomando como principal referencia sus narrativas. El enfoque adoptado para el desarrollo de la investigación articuló aportes de la teoría foucaultiana, la teoría de género y los estudios sociales de la discapacidad o disability studies en un intento por minimizar los sesgos sexistas y discapacitistas (Cobeñas, 2018). A partir de las perspectivas teóricas asumidas, se orientaron los esfuerzos a intentar dar cuenta del punto de vista de las y los jóvenes sobre los procesos de inclusión y exclusión educativa en los que estaban implicadas/os.

La elección del campo metodológico tiene su origen en el posicionamiento de los enfoques adoptados. Nos ubicamos así, en la perspectiva de investigación feminista sobre la discapacidad, (Cobeñas, 2018) que articula los enfoques de la investigación emancipatoria (Oliver, 2008; Susinos y Parrilla, 2008) y la investigación feminista (Harding, 2002). Este enfoque guio las decisiones en términos de métodos y formas de análisis. Así, se definió seleccionar como parte de la muestra a mujeres con diversos tipos de discapacidad, intentando escapar las definiciones de la discapacidad basadas en el modelo médico, pero articulando con las clasificaciones escolares. Seleccionamos así en un inicio a seis escuelas a las que asistieran jóvenes mujeres con discapacidad considerando los siguientes criterios: que pertenecieran tanto a sectores populares como a clase media, para intentar evitar el sesgo de clase; que tuvieran entre 15 y 29 años, ya que en un estudio previo identificamos que éste es el rango etario en el que se presenta la menor escolarización del conjunto de mujeres con discapacidad en la provincia de Buenos Aires (Cobeñas, 2015); que representaran diferentes tipos de discapacidad, específicamente las mujeres con discapacidades múltiples y profundas, quienes fueron identificadas como las más vulneradas en su derecho a la educación por el mismo estudio (Cobeñas, 2015). Asimismo, producto de la perspectiva metodológica asumida, definimos en un segundo momento incluir a dos mujeres con discapacidad activistas, para incorporar la voz de mujeres que estuvieran dialogando con espacios de construcción colectiva y política de la discapacidad 
para acceder a los puntos de vista de las jóvenes involucradas en el movimiento social de personas con discapacidad.

Para ello, un primer paso consistió en solicitar a las autoridades educativas que nos faciliten el dato de las escuelas a donde asistían jóvenes con estas características. La definición de cuáles y cuántas escuelas estuvo dada así por las autoridades del sistema educativo provincial, aunque solicitamos que se incluyeran escuelas tanto especiales como comunes. Se definieron las siguientes instituciones: cuatro escuelas de educación especial (para personas con discapacidad motriz, para personas con discapacidad intelectual, para personas ciegas y disminuidos visuales y para "multi-impedidos") y cuatro escuelas de educación común.

En dicho marco entrevistamos y observamos en contexto escolar a ocho mujeres con discapacidad entre 15 y 29 años que estuvieran o hubieran asistido a escuelas comunes (Cobeñas, 2015). Asimismo, hemos realizado entrevistas a jóvenes varones con discapacidad, familiares y a personal escolar como equipo directivo, equipo de orientación escolar, trabajadoras sociales y docentes entre otras. Si bien en nuestro estudio pusimos el foco en las voces de las personas con discapacidad, en el presente artículo incluimos múltiples referencias a las voces de los directivos, docentes y otros agentes ya que nuestro objetivo es describir las formas de exclusión educativa al mismo tiempo que los supuestos pedagógicos que hay por detrás, los cuales entendemos posibles aprehender a través de dichas voces.

El trabajo de campo se realizó en dos etapas, ambas en escuelas de gestión estatal de la provincia de Buenos Aires, Argentina, que atienden a sectores populares y de clases medias. La metodología utilizada para el trabajo en las escuelas fue cualitativa y hemos utilizado métodos etnográficos que consideramos adecuados para la recolección de datos en cuatro escuelas comunes secundarias y cuatro escuelas especiales que visitamos durante dos períodos continuo ocho meses cada uno, con una frecuencia de entre dos y tres días semanales y de dos a cuatro horas cada día. Así, hemos realizado observaciones participantes y no participantes, así como entrevistas semi-estructuradas e informales (Guber, 2011), debido a que responden a nuestro interés de acceder a los sentidos que los sujetos les dan a sus acciones y sus formas de auto percibirse en el contexto específico escolar (Guber, 2011; Rockwell, 2009). Asimismo, hemos desarrollado un análisis etnográfico de los datos, que implica una tarea constante de revisión y (re)construcción de los datos previo y durante el trabajo de campo, identificación y construcción de categorías teóricas y nativas y re elaboración de los dispositivos de observación y entrevista, propuesta por los enfoques socio-educativos antropológicos (Rockwell, 2009).

Si bien nuestro estudio estuvo orientado a describir las formas de exclusión que afectan específicamente a las jóvenes mujeres con discapacidad, en el desarrollo del estudio pudimos encontrar formas que afectan tanto a mujeres como varones con discapacidad. En el presente artículo proponemos dar cuenta de estos últimos resultados.

Por último, hemos incluido el análisis de normativa nacional y provincial que regula el sistema educativo a través de métodos documentales. 


\section{Resultados: Acerca de los distintos tipos de incumplimiento al derecho a la educación}

Nuestro estudio tuvo como objetivo central problematizar los procesos de inclusión y exclusión educativa de las personas con discapacidad en general y de las mujeres en particular. Consideramos que la relevancia del mismo radica en que describe y analiza las formas de exclusión actualmente existentes en Argentina, desde las perspectivas de las narrativas de las personas con discapacidad.

A continuación, desarrollaremos entonces los principales resultados de nuestro estudio, intentando recuperar tanto los criterios pedagógicos que subyacen a las diversas formas en que se expresa la exclusión educativa, así como sus supuestos y efectos desde las voces de los diversos actores educativos, tomando como voz privilegiada la del alumnado con discapacidad.

\subsection{Exclusión de la educación escolar}

Desde una perspectiva pedagógica, por exclusión escolar entendemos la negación de la posibilidad de educar a una persona por considerar que posee un déficit, una característica individual que lo/la imposibilita para el aprendizaje. En esta línea, la discapacidad es comprendida como una falla orgánica, desde una perspectiva biologicista y esencialista, como un dato objetivo e inalterable de la realidad, que torna al sujeto que la porta en ineducable. Esta forma de pensar la discapacidad es comprendida dentro del paradigma del modelo médico ya desarrollado. Así, hemos observado que ese criterio pedagógico produce que las escuelas dividan el alumnado entre educables e in-educables, siendo las personas con discapacidad y dentro de ellas las personas con discapacidades múltiples y profundas asociadas directamente con la in-educabilidad (Cobeñas, 2015).

Si bien las leyes argentinas de educación nacional y provincial vigentes y la Convención sobre los Derechos de las Personas con Discapacidad prohíben excluir a los niños de la posibilidad de recibir educación, esto aún sucede para el grupo de las personas con discapacidad, a quienes se les excluye de la escuela común, y en ocasiones también se les excluye de la escuela especial mediante el supuesto de que su deficiencia es tal que no pueden aprender. Como ejemplo de esta situación, un joven con discapacidades múltiples explica:

En mi infancia, mi vida estuvo muy condicionada por las decisiones de mi escuela especial. Me tuvieron en la escuela sin darme nada de educación, hasta que me dejaron fuera de ella. Entrar y permanecer en la escuela especial es muy dificil cuando piensan: "este chico es in-educable. (Entrevista no estructurada a un joven con discapacidad activista usuario de Comunicación Aumentativa Alternativa)

Así, las exclusiones, producidas bajo un criterio pedagógico que sostiene que las personas con discapacidad no pueden aprender, y que por ello no hay posibilidad de educarlas, se pueden observar en escuelas comunes, así como también en especiales.

En el caso de las escuelas comunes, encontramos que es una práctica extendida la eximición de alumnos con discapacidad de ciertas materias, o bien la derivación a escuelas de educación especial, legitimadas con el argumento de que la complejidad del modo de aprendizaje del alumno no puede ser abordado por la escuela común y hasta en ocasiones esgrimiendo que ya se ha intentado todo pero que "ese alumno no aprende". En ninguna de las entrevistas con personal docente de escuelas comunes describieron con claridad las estrategias pedagógicas que habían sido utilizadas. Por el contrario, corrieron el foco en 
describir las imposibilidades de aprendizaje del alumnado con discapacidad en el vacío, descontextualizado de toda referencia a procesos de enseñanza. Esto nos permite hipotetizar que lo que produce la exclusión es una idea de la discapacidad como un déficit individual que impediría que los alumnos aprendan, independientemente de los procesos de enseñanza. Esto resulta grave, ya que produce prácticas de negación de la educación, o sea, de exclusión.

Un dato relevante es que no hemos identificado la asistencia de alumnos/as con discapacidades múltiples, severas o profundas, especialmente aquellos que no se comunican convencionalmente, en escuelas comunes públicas de la provincia de Buenos Aires. Cuando indagamos con autoridades y docentes de las escuelas especiales acerca de la ausencia de estos grupos de alumnos/as en la escuela común han respondido que no pueden ser integrados/as, sin ser explícitos en los argumentos que fundan esta exclusión. Esto nos hace pensar que lo que define su integrabilidad es la misma condición de discapacidad. Esto resulta un criterio discriminatorio y discapacitante. Además, el sistema educativo clasifica entre sus escuelas especiales a aquellas que solamente atienden a niños, niñas y jóvenes con dos o más discapacidades, denominados "multi-impedidos" por el sistema educativo. Esta forma de denominación escolar nos resulta interesante ya que permite interpretar con relativa transparencia la concepción del sistema educativo sobre la discapacidad. Es decir, una mirada desde el modelo médico, considerando que los niños se encuentran impedidos por sus características. Contra esa mirada, nuestra perspectiva, desde el modelo social postula que dichos niños se encuentran impedidos de formas múltiples por el sistema educativo en el pleno ejercicio de múltiples derechos, incluido el de la educación. En referencia al caso de los/as jóvenes alumnas/os "multi-impedidos/as", una maestra especial nos explicó sus destinos escolares posibles.

Hay talleres laborales protegidos integrales para los chicos que tienen una discapacidad para hacer un oficio, y los que son más severos se quedan en la escuela ya sin la parte pedagógica, que es la que te da el integral que tiene contenidos de secundaria. (Entrevista con docente de escuela especial para personas con discapacidad motriz)

Por otro lado, un integrante del personal de otra escuela especial describía así la dinámica de escolarización de los grupos de personas con discapacidades múltiples que asisten a una escuela especial para personas con discapacidad motriz:

\section{A la tarde tenéis los chicos más comprometidos, que vienen a la escuela a recibir estímulo solamente porque no pueden acceder a lo pedagógico. (Entrevista informal con trabajadora social, escuela de educación especial para personas con discapacidad motriz)}

Es decir, si bien los alumnos asisten a la escuela especial, la institución no considera que lo hacen para educarse, por lo que podemos identificar esta situación como de exclusión. Aquí, la escuela especial oficia de centro de día para estos niños, niñas y jóvenes que no son considerados educables, ya que sólo se los considera aptos para prácticas de estimulación y rehabilitación. La posibilidad de acceder a "lo pedagógico" divide a las personas con discapacidad a través de un discurso de saber que identificará no sólo quienes están o no capacitados para acceder a la escuela común al mismo tiempo que señalará quiénes podrían o no recibir una educación especial. De este modo, lo pedagógico se constituye en un conjunto de prácticas y saberes que involucra el reconocimiento de capacidades intelectuales o motrices para su realización. Esto resulta en que los y las niños/as y jóvenes con discapacidades múltiples y profundas no califiquen para ser 
educados y sean sistemáticamente excluidos de toda forma de educación. Tal como afirmó la directora de una escuela especial para "multi-impedidos":

En mi escuela ahora me dicen que no puede ser que tenemos el 100\% de los alumnos analfabetos. Lo que pasa es que antes la escuela especial era para cuidar y ayudar a los alumnos, no sabíamos que también los teníamos que alfabetizar, sobre todo con las características de mis alumnos, que no pueden ser alfabetizados. (Entrevista semiestructurada con directora de escuela especial para "multi-impedidos")

Según esta directora, al momento de la entrevista, la totalidad del alumnado de su escuela era analfabeto porque entiende que no pueden aprender ningún sistema de lectoescritura o de comunicación. Asimismo, la directora describe la función de la escuela especial como un lugar de cuidado o de asistencia, pero no de educación. es decir que las escuelas especiales no tienen como principal objetivo la enseñanza. Esto resulta llamativo y parece contrastar con la imagen social de la función que debe cumplir una institución educativa. Asimismo, hemos registrado que docentes de educación común derivan a alumnos con discapacidad a escuelas especiales por pensar que en dicho espacio circulan docentes con conocimientos didácticos con alguna especificidad asociada a las formas de aprendizaje de las personas con discapacidad. Sin embargo, la definición de esta directora parece contradecir esa expectativa, además de todos los estudios sobre educación inclusiva que sostienen que todos los niños aprenden con los mismos enfoques didácticos y deben hacerlo en entornos inclusivos.

De modo que en las escuelas especiales no solo se excluye negando la enseñanza, sino que se violan otros derechos, como por ejemplo a disponer de un sistema de comunicación acorde a sus requerimientos: los y las alumnos/as de esa escuela están incomunicados/as porque por definición son consideradas como personas incapaces de comunicarse.

Hemos podido conversar con jóvenes con discapacidad que forman o formaron parte del grupo de "no integrables" y que, fuera de la escuela, pudieron acceder a sistemas de comunicación alternativos aumentativos. En sus narrativas reproducen con dolor, malestar y angustia los comentarios denigrantes que las maestras de escuelas especiales realizaron constantemente en su presencia, dado que ignoraban el hecho de que esos alumnos podían comprenderlas aun cuando ellas actuaban como si no estuvieran allí. Resaltan que estas experiencias los/las han marcado profundamente incidiendo en visiones negativas de sí, siendo atravesados por sentimientos de temor y angustia cuando se encontraban frente a situaciones educativas. Según un joven activista por los derechos de las personas con discapacidad y usuario de comunicación alternativa aumentativa:

$$
\begin{aligned}
& \text { P: Yo puedo acceder al mundo tuyo a través de mi comunicador. Puedo ser visto y } \\
& \text { oído gracias a él. Por eso es parte de mí como tu voz para vos. Y sin él podría estar, } \\
& \text { pero sería muy... no me lo puedo imaginar. No recuerdo mi vida sin él. } \\
& \text { E: ¿̇ qué pasa con la silla de ruedas que también te ayuda a moverte? } \\
& \text { P. Podría no tenerla y la extrañaría. Para mí es muy importante, pero no tanto como } \\
& \text { el comunicador. Porque es mi voz y mi forma de hacerme ver. Sin él no me ve ni mi } \\
& \text { familia ni nadie. (Entrevista no estructurada a un joven con discapacidad } \\
& \text { activista usuario de Comunicación Aumentativa Alternativa) }
\end{aligned}
$$

Por otro lado, en las escuelas especiales se observa un dispositivo de normalización que identificamos como un modo sistemático de exclusión que constituye una forma de opresión como marginación es la de la incorporación de alumnos y alumnas a centros de día esgrimiendo que no pueden aprender tampoco en la escuela especial. Estos alumnos asisten a centros de día que se definen como: 
(...) una institución destinada a recibir a jóvenes y/o adultos discapacitados severos y profundos, en situación de dependencia, egresados de la escuela especial, sin posibilidades de acceder al sistema laboral protegido; y/o a niños que por las características de su discapacidad no pueden acceder a las currículas contempladas en el área de Educación Especial. (IOMA, resolución 3624/10, anexo I)

Para ser excluido/a de la escuela especial e ingresar en un centro de día, según IOMA, la obra social de la provincia de Buenos Aires que cubre los gastos del servicio, los requisitos son:

Derivación Médica, Certificado de Discapacidad, emitido por organismo oficial. Baja escolar (para alta en Centro de Día), o Informe del equipo de la Escuela Especial (para alta en CET). Informe de admisión del Centro con plan de tratamiento $y$ presupuesto. (IOMA, resolución 3624/10, anexo II)

De acuerdo a los requisitos para declarar a alguien como in-educable, se precisa un diagnóstico médico y una baja de la escuela. Es entonces un saber medico pedagógico el que actúa declarando que unas infancias y juventudes carecen de toda posibilidad de ser educados y trabajar, y definen un destino institucional que los sepultará de toda posibilidad de constituirse como sujetos de educación, derecho y vida independiente.

\subsection{Segregación en escuelas especiales y talleres laborales protegidos}

Hemos encontrado que otra forma de violación de los derechos de las personas con discapacidad es la segregación escolar. Esto implica ubicar al/la alumno/a en las escuelas especiales bajo el supuesto, legitimado por los diagnósticos médico-psiquiátricospsicológicos-pedagógicos, de que no puede asistir a la escuela común a causa de sus deficiencias. Desde esta perspectiva, son los alumnos los que deben reunir ciertas características para poder ser educados en contextos "comunes". Aquellos que no cumplan con ciertos requisitos deben ser separados del resto de alumnos y agrupados en instituciones que se clasifican por tipo de discapacidad.

Así, mientras los agrupamientos de los niños y niñas sin discapacidad se organizan en niveles educativos y edades (gradualidad), la clasificación escolar para los niños con discapacidad es por tipo de discapacidad. Esta forma educativa se basa en perspectivas médico-pedagógicas que suponen que son los niños los que deben adecuarse al sistema educativo y no a la inversa, apoyándose en criterios pedagógicos normalizadores que parten de suponer que todos los niños aprenden lo mismo de la misma forma a la misma edad.

Si bien la Convención prohíbe cualquier forma de educación segregada, la escuela especial como espacio institucional discriminatorio subsiste de forma eufemizada. Así, el marco normativo que debería transformar la escuela especial en una de las formas de apoyo a la inclusión es ambiguo en su expresión de las funciones de la escuela especial. Si bien expresa que esta es una modalidad del sistema de educación común y no un subsistema segregante, expresa en los mismos documentos que la escuela especial retendrá en sus sedes a aquellos alumnos que no puedan asistir a escuela común a causa de sus características. Es decir, mediante el eufemismo de modalidad se esconde un sistema de segregación escolar destinado a los alumnos "anormales" que se mantiene intacto.

La mayoría de los miembros de los equipos docentes y de gestión de las escuelas especiales estudiadas defienden una educación segregada basada en diferentes diagnósticos, caracterización de impedimentos o patologías que agrupan a alumno/as. Por ejemplo, en 
una escuela especial para personas con discapacidad intelectual, la directora definió al alumnado de la siguiente manera:

La matrícula de la escuela se divide según la patología. En general el $80 \%$ de la matricula son repitentes, por ejemplo, tienen 12 años y están en $2^{\circ}$ grado. Tienen un déficit por la situación social, por lo que deviene de la situación social y económica y sus carencias. El otro 20\% tiene algún diagnóstico genético, alguna discapacidad, atención temprana. (Entrevista semi-estructurada a directora de una escuela especial para personas con discapacidad intelectual)

Entre el personal de las escuelas especiales entrevistadas, hemos observado una evidente resistencia a la inclusión de personas con discapacidad en escuelas comunes y esto se expresa y realiza de diversas maneras. Los docentes y directivos de instituciones educativas especiales sienten como una intromisión y, en ocasiones, un ataque, al reclamo de las familias de integrar a sus hijos/as en escuelas comunes. La directora de una escuela especial para personas con discapacidad intelectual señaló que un 20\% de su matrícula se encontraba integrada en escuelas comunes y agregó que,

cuando los padres piden que se los integre se les pide que busquen una escuela común. Está instalado que es genético y que la sociedad debe aceptarlos, pero es peor, y a medida que crecen es peor [para los y las alumnos/as con discapacidad] estar en la escuela común. Se generan, entre otros, problemas de autoestima que vienen con la adaptación al maestro integrador. Así, cuando se ve que una secundaria no va a ayudar se decide que continúe en sede [escuela especial] para hacer formación laboral. (Entrevista semi-estructurada a directora de una escuela especial para personas con discapacidad intelectual)

Al mismo tiempo, nos interiorizamos, a partir de entrevistas, acerca de la visión de personas con discapacidad y sus familias y de representantes de las diferentes organizaciones que las nuclean, principalmente en la región bonaerense, quienes manifiestan que es muy común que las escuelas especiales les recomienden que su hijo/a no vaya a escuela común proponiendo como argumento principal que "es lo mejor para el niño o la niña”. También es frecuente que exhorten a las familias para que el/la alumno/a con discapacidad que está integrado/a en una escuela común retorne a la escuela especial. En todos los casos, el personal docente o equipo directivo de escuelas especiales justifican sus decisiones sobre la base del conocimiento experto de docentes especiales, terapistas ocupacionales, psicopedagogos, fonoaudiólogos, psicólogos, médicos, y demás profesionales de la escuela especial. Hemos registrado que durante dichos procesos resulta común que familias, niños, niñas y jóvenes con discapacidad expresen insistentemente su deseo de no cambiar de grupo o de salir de la escuela común para ir a escuelas especiales, pero que raramente esas opiniones son tenidas en cuenta por el equipo de gestión y docente de las escuelas comunes o especiales. En este sentido, una directora de escuela especial señala que:

Cuando los chicos son grandes y terminan $6^{\circ}$ se reúne a la escuela con la familia, y el alumno para definir si va a la escuela secundaria o al CFI [Centro de Formación Integral]. Los chicos nunca quieren ir al CFI, pero es lo mejor para ellos. (Entrevista semi-estructurada a directora de una escuela especial para personas con discapacidad intelectual)

Asimismo, una maestra integradora expresó que,

Los padres son muy sobreprotectores, siempre están de acuerdo con ir a común, en general se resisten a especial. (Entrevista semi-estructurada a una docente de una escuela especial para ciegos y disminuidos visuales) 
Como contraste, resulta significativo recuperar las voces de las personas con discapacidad en torno a su experiencia en escuela especiales. Por ejemplo, Tara Flood (2004), una mujer británica con discapacidad activista refiere a su experiencia en la escuela especial de la siguiente manera:

\begin{abstract}
En lo personal, soy sobreviviente de una educación segregada y siempre tuve la esperanza de que mi generación de niños con discapacidad sea la última en experimentar la educación "segregada", pero lamentablemente esto no sucede. /.../ Desafortunadamente para mí, carezco de paciencia y siempre me sentí frustrada debido a que las cuestiones no avanzan con mayor rapidez. Puedo aceptar que los vientos de cambio soplen extremadamente despacio, pero ver cómo los niños con discapacidad experimentan la misma actitud de aislamiento, exclusión y deshumanización que mi generación es inaceptable en un mundo que se considera civilizado (p. 1)
\end{abstract}

En el cuadro 1 mostramos las trayectorias educativas posibles para un/a alumno/a que ha sido etiquetado como con discapacidad en algún momento del proceso educativo, según la normativa educativa de la provincia de Buenos Aires.

Cuadro 1. Trayectorias educativas para personas con discapacidad según Resolución 1269/11 de la DGCyE, Prov. De Bs. As. Argentina

\begin{tabular}{|c|c|c|c|}
\hline \multirow{2}{*}{$\begin{array}{c}\text { NivEL } \\
\text { EDUCATIVO }\end{array}$} & \multirow[b]{2}{*}{ EDAD (AÑOS) } & \multicolumn{2}{|c|}{ TIPO DE ESCOLARIZACIÓN } \\
\hline & & $\begin{array}{c}\text { En Sede (en escuelas } \\
\text { especiales) }\end{array}$ & $\begin{array}{c}\text { Con Proyecto de Integración } \\
\text { (en escuelas comunes) }\end{array}$ \\
\hline \multirow{2}{*}{ Inicial } & o a 3 & $\begin{array}{c}\text { Centro de Atención } \\
\text { Temprana del } \\
\text { Desarrollo Infantil } \\
\end{array}$ & Jardín Maternal \\
\hline & $\begin{array}{c}3 \text { a } 5 \\
\text { (hasta } 7 \text { máx.) }\end{array}$ & Escuela Especial (sede) & Jardín de Infantes \\
\hline Primario & $\begin{array}{c}6 \text { a } 14 \\
(14 \text { máx.) }\end{array}$ & $\begin{array}{l}\text { Escuela Especial (sede) }+ \\
\text { Formación Laboral }\end{array}$ & $\begin{array}{l}\text { Escuela Primaria Común } \\
\text { Escuela Primaria Común }+ \\
\text { Escuela Especial (sede) }+ \\
\text { Formación Laboral } \\
\end{array}$ \\
\hline Secundario & $\begin{array}{c}14 \text { a } 21 \\
\text { (21 máx.) }\end{array}$ & $\begin{array}{l}\text { Centros de Formación } \\
\text { Integral para } \\
\text { adolescentes, jóvenes y } \\
\text { adultos con } \\
\text { discapacidad }\end{array}$ & $\begin{array}{l}\text { Escuela Secundaria Común } \\
\text { Escuela Secundaria Común }+ \\
\text { Centros de Formación } \\
\text { Integral para adolescentes, } \\
\text { jóvenes y adultos con } \\
\text { discapacidad }\end{array}$ \\
\hline
\end{tabular}

Fuente: Elaboración propia a partir de la Resolución 1269/11 DGCyE.

La resolución $\mathrm{N}^{\circ}$ 1269/11 de la Dirección General de Cultura y Educación indica que los pasajes de una escuela común a una escuela perteneciente a la modalidad de educación especial se pueden dar inicialmente en tres momentos del año que corresponden a tres secciones divididas por informes de evaluación del alumnado con discapacidad. Esta evaluación según se indica en la resolución, es realizada por un equipo transdisciplinar, quien indicará al finalizar cada trimestre y cada año lectivo si el alumno con discapacidad puede continuar su escolaridad en el sistema común o deberá ser derivado de éste para asistir a las escuelas especiales de la modalidad. El criterio para escolarizar al alumnado en escuelas segregadas es, según la resolución, "cuando la complejidad lo amerite". Esto resulta una situación de continua evaluación sobre el alumnado con discapacidad y una suerte de prueba o test constante en el que deben demostrar que son dignos de permanecer en la escuela común. Al mismo tiempo, debemos considerar que la cantidad de horas que 
una maestra integradora comparte con el/la alumno/a con discapacidad en la escuela común es de alrededor de dos horas por semana. Es imposible pensar que se pueda evaluar el desempeño de un alumno en ese corto período de tiempo. Sin embargo, sí podemos evaluar la posibilidad de incidencia de la maestra integradora ateniéndonos a la pobre carga horaria compartida con el alumnado, hipotetizando que será, en muchos casos pobre o nula.

Como se puede ver en el cuadro, según la resolución 1269/11, aquellos alumnos que, "a causa de su discapacidad” no puedan acceder a la escuela común, asistirán puramente a escuelas especiales o sus distintos centros de formación laboral. Esta derivación puede suceder en cualquier momento de la escolarización.

Los alumnos que hayan cumplido con el nivel de educación primaria y que por la complejidad de sus necesidades educativas no puedan concurrir a la escuela del nivel secundario recibirán una propuesta pedagógica individualizada en la sede de las instituciones de Educación Especial que, a partir de la presente Resolución, se desarrollarán en el marco de los Centros de Formación Integral de Adolescentes, jóvenes y adultos con discapacidad. (Res. 1269/11 de la DGCyE)

El término "complejidad de sus necesidades educativas" (NE) es de tal modo arbitrario que su impresión y su carácter universalizante a la vez que particular deja en manos de aquellos que están a cargo de los procesos educativos y de aquellos que ejecutan los procedimientos administrativos las decisiones acerca de quiénes serán investidos con esa nominación. Así, es a través de este tipo de discurso basado en clasificaciones no referenciadas en un campo de saber específico como "profundo", "complejo", "leve", "moderado", que un equipo de profesionales define los destinos escolares de las personas con discapacidad, donde la posibilidad de escolarización en escuela especial sin integración es una de las opciones.

En términos didácticos, hemos podido registrar como producto de las observaciones de clases que las docentes raramente organizan las actividades basadas en el Diseño Curricular correspondiente. Más bien, utilizan como fuente de actividades propuestas que circulan en internet, pero que no responden a objetivos o contenidos pedagógicos contextualizados, enlazados ni planificados. Tampoco ponen en juego estrategias de enseñanza específicas ni brindan apoyo a sus alumnas para que desarrollen la actividad. El día escolar se compone así de células desconectadas de actividades que responden de forma dudosa al contenido previsto en el diseño curricular (Cobeñas, 2016). Sumado a ello, en un estudio sobre la enseñanza de la matemática en escuelas especiales que estamos desarrollando, hemos podido convalidar la hipótesis de que las clases guardan escasa o nula referencia con los Diseños Curriculares. Asimismo, también encontramos que el alumnado tiene escaso nivel de participación y prevalecen intercambios en los cuales hay escasa presencia de trabajo matemático. Hemos identificado que se reproducen condiciones de exclusión y un círculo vicioso de enseñanza con bajo nivel de expectativas, sumado a la pervivencia de ideas de perspectivas obsoletas sobre la enseñanza (Broitman et al., 2017, 2018).

\subsection{Integración: Exclusión en la inclusión}

Hemos registrado múltiples situaciones en las que los y las alumnos/as con discapacidad sufren tratos violentos, subalternizantes, inferiorizantes, discapacitantes, de modo que queremos señalar enfáticamente que no alcanza con permitir que las y los jóvenes con discapacidad asistan a la escuela común para denominar a esa práctica como educación inclusiva. Es transformando las escuelas y sus agentes para poder alojar a ese alumnado 
en sus aulas que se garantizarán plenamente sus derechos y, de ese modo, se evitará que continúen atravesando por situaciones de violencia cultural, simbólica y hasta física.

Entre los obstáculos a la plena inclusión hemos registrado malos tratos, eximición de clases o materias en escuelas comunes, prescripción de permanencias y ausencia de apoyos. Entre ellos, una alumna joven con discapacidad, quien debió continuar sus estudios en una escuela para adultos luego de repetir cuatro años seguidos en su escuela común la materia matemática, nos relató una situación con una profesora de esa misma materia en su escuela actual:

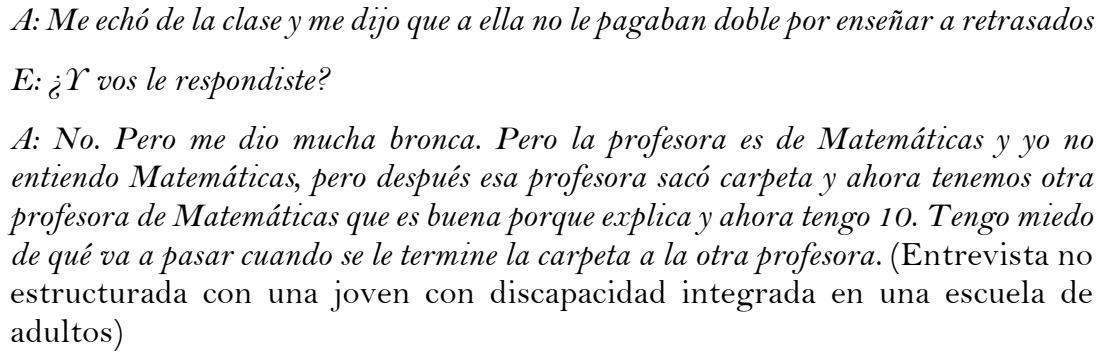

El término "retrasado" que utiliza la docente supone una concepción que estableció la pedagogía normalizadora acerca de que es un alumno normal, o sea, quien puede habitar la escuela, y quien no, basado en una didáctica tradicional que solo reconoce una forma de enseñar y que supone que todos aprenderán al mismo tiempo del mismo modo. Esta situación también demuestra como siguen siendo los docentes la voz de alarma de la anormalidad de la alumna. Por otro lado, el maltrato y la violencia de la docente son posibles debido al no reconocimiento de la alumna como sujeto de educación ni de derecho.

Si en una escuela común se inscribe una persona con discapacidad, dicha escuela se vincula a una escuela especial para acordar un proyecto de integración en el que se prevé entre otros, la designación de una docente integradora de la escuela especial que acompañará durante dos horas reloj por semana a la alumna o el alumno dentro del aula. Esa docente, quien comparte en el mejor de los casos dos horas semanales con el alumno, es la que la evaluará y más tarde decidirá si ese niño o joven puede continuar en la escuela común o debe ser derivado a una escuela especial. Asimismo, el proyecto de integración es producido por el equipo interdisciplinario de la modalidad especial con base en el legajo y documentos provistos por la institución escolar común, así como en función de conversaciones formales e informales con docentes y equipo directivo y de orientación educativa de esa institución. De modo que dicho proyecto pedagógico es producido sin ni siquiera haber visto o interactuado con el alumnado en la mayoría de los casos. Sumado a esto, las escuelas especiales no proveen apoyos a las personas con discapacidad que asisten a las escuelas comunes, solo proveen una maestra integradora sin formación disciplinar (didácticas específicas) ni en la enseñanza en cada nivel educativo (inicial, primario, secundario).

Resulta necesario insistir en que la educación inclusiva no significa la mera presencia física de las personas con discapacidad en la escuela común. Así, es preciso partir de diferenciar la integración de la educación inclusiva. La integración supone que son las personas con discapacidad las que deben adaptarse a las instituciones escolares comunes para ser consideradas como alumnas legítimas. De esta forma, las instituciones no ofrecen recursos ni re estructuraciones de ningún tipo. Y los recursos que se ofrecen parten desde un modelo rehabilitatorio-normalizador en la medida en que parten de considerar que son los alumnos los que tienen los problemas, los déficits y las limitaciones. De este modo, es muy 
común que ni la institución en su conjunto ni los docentes de educación común crean que el alumno con discapacidad sea su alumno legítimo y los docentes de educación especial encuentren dificultades varias en su tarea como integradores. Por ejemplo, la escasa cantidad de horas que asisten a las escuelas comunes, la insuficiente formación en las didácticas específicas y de niveles, entre otras (Cobeñas, 2016). En conclusión, el alumnado con discapacidad recibe tareas diferenciadas, desde enfoques didácticos diferenciados, en ocasiones en espacios diferenciados e incluso con docentes diferenciados.

Sumado a esto, como figuras de apoyo se encuentran otros perfiles no docentes, que son financiados y formados por áreas dependientes del ministerio de salud, tales como los acompañantes terapéuticos. Hemos registrado extensamente casos donde los acompañantes terapéuticos sostienen la función docente debido a que las instituciones de educación común no reconocen a la persona con discapacidad como estudiante legítimo y no se responsabilizan de los procesos de enseñanza (Cobeñas y Grimaldi, 2018), con consecuencias pedagógicas tales como la devaluación del contenido o el efectivo fracaso escolar, asignado a los déficits del alumnado con discapacidad y no a las falencias del sistema educativo. De modo que, las funciones pedagógicas quedan en manos de la buena voluntad de las personas que acompañan o asisten a los alumnos con discapacidad, en ocasiones sin formación pedagógica. Sin embargo, aquellos alumnos provenientes de sectores populares no cuentan con esta posibilidad, por lo que consideramos que la negación de oportunidades se da con mayor fuerza en alumnos pertenecientes a dichos sectores, lo cual intensifica la desigualdad.

\section{Conclusiones}

En nuestro estudio pudimos identificar al menos tres grados de incumplimiento del derecho a la educación educativa en la escolarización de las personas con discapacidad: exclusión escolar, segregación e integración o exclusión en la inclusión. Estas categorías de análisis las recuperamos tanto del área de estudios académicos sobre educación inclusiva (Ainscow, 1998, 2002; Barton, 1998; Echeita, 2008; Parrilla, 2002; Porter, 2008; Rappoport et al., 2019), así como de los documentos de derechos humanos provenientes de las Naciones Unidas al respecto. Así, tal como explica el Estudio Temático (2013) acerca del derecho de las personas con discapacidad a la educación:

En la Convención sobre los derechos de las personas con discapacidad se señala que, para que estas personas puedan ejercer ese derecho, han de existir sistemas educativos inclusivos; en consecuencia, el derecho a la educación es un derecho a la educación inclusiva.

Por lo general, los sistemas de enseñanza han adoptado uno de los tres enfoques siguientes con respecto a las personas con discapacidad: la exclusión, la segregación y la integración. La exclusión se produce cuando se mantiene apartado a un estudiante de la escuela debido a la existencia de una deficiencia, sin que se le ofrezca otra opción educativa en pie de igualdad con los demás estudiantes. (...). La segregación tiene lugar cuando un estudiante con esas características es remitido a un centro educativo diseñado específicamente para responder a una deficiencia concreta, normalmente en un sistema de enseñanza especial. Por último, la integración consiste en que los alumnos con una deficiencia asistan a una escuela convencional, mientras puedan adaptarse y cumplir los requisitos normalizados del centro docente. El enfoque de la integración se centra exclusivamente en reforzar la capacidad del estudiante para cumplir las normas establecidas. (pp. 3-4) 
Desde estos dos campos es que hemos podido clasificar y definir las formas de exclusión educativa que afectan específicamente a las personas con discapacidad en Argentina. Intentamos en nuestra clasificación identificar los criterios pedagógicos que subyacen a cada forma de exclusión específica.

Podemos afirmar que en las escuelas argentinas de la provincia bonaerense se sostienen prácticas, culturas y políticas que producen diferentes formas de exclusión educativa en general (exclusión propiamente dicha, segregación e integración) que viola el derecho a la educación de las personas con discapacidad. Asimismo, hemos encontrado que en todas las formas subyacen supuestos pedagógicos que ponen énfasis en las imposibilidades del alumnado y que niegan o devalúan los procesos de enseñanza, al identificar y clasificar el estudiantado en educable e in-educable. Esto estaría basado en una concepción desde el paradigma del modelo médico de la discapacidad. Desde el paradigma del modelo social de la discapacidad, entendemos que la educación solo puede ser inclusiva para asegurar el pleno ejercicio del derecho a la educación, y que son las escuelas las que deben transformarse de acuerdo a las características de los alumnos y no los alumnos los que deben cumplir con ciertos requisitos para ser considerados educables y en entornos comunes. Así, hemos desarrollado nuestro estudio con la intención de contribuir a profundizar la mirada sobre las condiciones pedagógicas para la transformación del sistema educativo argentino en inclusivo en el marco del proceso de cumplimiento (o incumplimiento) de los derechos consagrados en la Convención.

Si bien el estudio, por sus características metodológicas, no permite establecer una generalización de los resultados, hemos intentado en estudios previos establecer un diálogo con los datos estadísticos existentes sobre la situación educativa de las personas con discapacidad y las mujeres en particular. Dada la escasez, irregularidad y poca confiabilidad de los datos (Cobeñas, 2015; Fara, 2010) consideramos que una interesante área de trabajo consiste en desarrollar estudios cuantitativos que permitan desarrollar la comprensión de los procesos de exclusión educativa que afectan específicamente a las personas con discapacidad en Argentina y, particularmente, en la provincia de Buenos Aires. Creemos que ello puede dar lugar además a extender estas relaciones en estudios que triangulen los datos cualitativos con los cualitativos. Asimismo, resultaría interesante replicar estudios similares tomando como sujetos de la investigación a niños y niñas con discapacidad en contextos urbanos y niños, niñas y jóvenes con discapacidad en contextos rurales, sobre lo que existen escasos avances.

Por otro lado, consideramos que nuestro estudio, así como futuros estudios sobre la misma línea de trabajo, pueden constituirse como investigaciones valiosas que puedan contribuir a desarrollar políticas públicas que partan de reconocer las barreras a la inclusión educativa de las personas con discapacidad en términos del modelo social y acordes con los acuerdos sedimentados en la Convención sobre los Derechos de las Personas con Discapacidad. Asimismo, esperamos que constituya una posibilidad de interpelación al campo pedagógico y de la investigación socio-educativa, para llamar la atención sobre la necesidad de construir conocimiento sobre la base de revisar los criterios discapacitistas que subyacen a las teorías y prácticas pedagógicas actuales en Argentina. En definitiva, hacer dialogar las investigaciones con las políticas públicas para que permitan problematizar las culturas, políticas y prácticas educativas actuales que perpetúan la exclusión de las personas con discapacidad, y con mayor fuerza, la de las mujeres con discapacidad. 


\section{Referencias}

Ainscow, M. (1998). Exploring links between special needs and school improvement. Support for Learning, 13(2), 70-75. https://doi.org/10.1111/1467-9604.00061

Ainscow, M. (2002). Rutas para el desarrollo de prácticas inclusivas en los sistemas educativos. Revista de Educación, 327, 69-82.

Barton, L. (1998). Discapacidad y sociedad. Madrid: Morata

Barton, L. (2009a). Estudios sobre discapacidad y la búsqueda de la inclusividad. Observaciones. Studies on disability and the quest for inclusivity: Some observations. Revista de Educación, 349, 137-152

Barton, L. (2009b). La posición de las personas con discapacidad. ¿Qué celebrar y por qué celebrarlo? ¿Cuáles son las consecuencias para los participantes? En P. Brogna (Comp.), Visiones y revisiones de la discapacidad. Ciudad de México: Fondo de Cultura Económica

Booth, T., Simón, C., Sandoval, M., Echeíta, G. y Muñoz, Y. (2015). Guía para la educación inclusiva. Promoviendo el aprendizaje y la participación en las escuelas: Nueva edición revisada y ampliada. REICE. Revista Iberoamericana sobre Calidad, Eficacia y Cambio en Educación, 13(3), 5-19.

Broitman, C., Cobeñas, P., Escobar, M., Grimaldi, V. (mayo, 2017). Enseñar y aprender matemática en aulas inclusivas. Comunicación presentada en el IV Seminario Nacional Red Estrado. Buenos Aires.

Broitman, C., Cobeñas, P., Divene, L., Escobar, M, Falco, L, González, E., Lemos, E., Miranda, L, Sancha, I., Goñi, M., Grimaldi, V. (septiembre, 2018) ¿Qué matemáticas escolares viven hoy en las aulas de educación especial? Comunicación presentada en las III Jornadas de Enseñanza, Capacitación e Investigación en Cs. Naturales y Matemática. Buenos Aires.

Cobeñas, P. (2014). Buenas prácticas inclusivas en la educación de personas con discapacidad en la provincia de Buenos Aires y desafíos pendientes. Buenos Aires: Asociación por los Derechos Civiles.

Cobeñas, P. (2015). Visiones de sí de jóvenes mujeres con discapacidad que asisten a escuelas públicas de la provincia de Buenos Aires. Tesis de maestría. Universidad Nacional de La Plata.

Cobeñas, P. (2016). Jóvenes mujeres con discapacidad en escuelas públicas de la provincia de Buenos Aires: problematizando los procesos de inclusión y exclusión educativa. Tesis de doctorado. Universidad Nacional de La Plata.

Cobeñas, P. (2018). Investigar con mujeres con discapacidad: Reflexiones epistemológicas y metodológicas desde el enfoque feminista-emancipador. Revista Brasileira de Estudos Pedagógicos, 99(251),132-147. https://doi.org/10.24109/2176-6681.rbep.99i251.3473

Cobeñas, P. y Grimaldi, V. (2018). Construyendo una educación inclusiva II. Aportes para repensar la enseñanza. La Plata: Asociación Azul.

Echeita, G. (2008). Inclusión y exclusión educativa: "Voz y quebranto". REICE. Revista Iberoamericana sobre Calidad, Eficacia y Cambio en Educación, 6(2), 9-18.

Echeita, G. y Ainscow, M. (2011). La educación inclusiva como derecho: Marco de referencia y pautas de acción para el desarrollo de una revolución pendiente. Tejuelo: Revista de Didáctica de la Lengua y la Literatura, 12, 24-46.

Fara, L. (2010) La población con discapacidad menor de 50 años en la Argentina: Una mirada desde la estadística. En C. Acuña y L. Bulit Goñi (Comps.), Políticas sobre discapacidad en la Argentina. El desafio de hacer realidad los derechos (pp. 73-124). Buenos Aires: Siglo XXI. 
Flood, T. (2004). Niños con discapacidad, la emergencia invisible. París: Alliance for Inclusive Education.

Guber, R. (2011). La etnografía: Método, campo y reflexividad. Buenos Aires: Siglo XXI.

Harding, S. (2002). ¿Existe un método de investigación feminista? En E. Bartra (Comp.), Debates en torno a una metodología feminista (pp. 9-34). Ciudad de México: PUEG-UAM.

Miles, S. y Ainscow, M. (2008). Por una educación para todos que sea inclusiva: ¿Hacia dónde vamos ahora? Perspectivas, 38(1), 17-45.

Morris, J. (1998). Feminism, gender and disability. Recuperado de http://www.leeds.ac.uk/disability-studies/archiveuk/morris/

Naciones Unidas. (2013). Informe anual. Estudio temático sobre el derecho de las personas con discapacidad a la educación. Recuperado de https://www.acnur.org/fileadmin/Documentos/BDL/2016/10350.pdf

Oliver, M. (2008) Políticas sociales y discapacidad. Algunas consideraciones teóricas. En L. Barton (Comp.), Superar las barreras de la discapacidad: 18 años de disability and society (pp. 19-33). Madrid: Morata.

Parrilla, A. (2002). Acerca del origen y sentido de la educación inclusiva. Revista de Educación, 327(1), 11-29.

Porter, G. L. (2008). Making Canadian schools inclusive: A call to action. Education CanadaToronto, 48(2), 62-66.

Rockwell, E. (2009). La experiencia etnográfica. Historia y cultura en los procesos educativos. Buenos Aires: Paidós.

Rappoport, S., Sandoval, M., Simón, C. y Echeita, G. (2019). Understanding inclusion support systems: Three inspiring experiences. Cultura y Educación, 31(1), 120-151. https://doi.org/10.1080/11356405.2019.1565250

Susinos, T. y Parrilla, A. (2008). Dar la voz en la investigación inclusiva. Debates sobre inclusión y exclusión desde un enfoque biográfico-narrativo. REICE. Revista Iberoamericana sobre Calidad, Eficacia y Cambio en Educación, 6(2), 157-171.

\section{Breve CV de la autora}

\section{Pilar Cobeñas}

Profesora y Licenciada en Ciencias de la Educación (Universidad Nacional de La Plata, Argentina). Es Magíster en Educación y Doctora en Ciencias de la Educación (UNLP, Argentina). Se desempeña como Jefe de Trabajos Prácticos de la cátedra Pedagogía de la Diversidad (UNLP) y ha realizado sus estudios de doctorado y posdoctorado como becaria del Consejo Nacional de Investigaciones Científicas y Técnicas (CONICET, Argentina). Es investigadora en el área de discapacidad, género y educación inclusiva. Actualmente se encuentra realizando estudios sobre la enseñanza de la matemática en aulas inclusivas. Es miembro activo de la Asociación Azul (ONG por los derechos de las personas con discapacidad) y co-coordina su Grupo de docentes. Ha dictado cursos de posgrado y de capacitación docente sobre discapacidad y educación inclusiva y es autora de diversas publicaciones sobre la temática. ORCID ID: https://orcid.org/OOOO-0003-0754-4628. Email:pilarcobenas@gmail.com 